\title{
Review
}

\section{Development of portable gas proportional scintillation counters for $x$-ray spectrometry ${ }^{\dagger}$}

\author{
J. M. F. dos Santos, ${ }^{*}$ J. A. M. Lopes, J. F. C. A. Veloso, P. C. P. S. Simões, T. H. V. T. Dias, \\ F. P. Santos, P. J. B. M. Rachinhas, L. F. Requicha Ferreira and C. A. N. Conde \\ GIAN-Departamento de Física, Universidade de Coimbra, P-3004-516 Coimbra, Portugal
}

Received 27 July 2000; Accepted 29 May 2001

\begin{abstract}
A review on gas proportional scintillation counters (GPSCs) is presented. Recent achievements towards the portability of simple, inexpensive and compact GPSCs are discussed. Compensation of solid angle effects with the curved grid technique can be used to produce non-focused GPSCs with medium-sized radiation windows, at least up to $80 \%$ of the photosensor active diameter, without degradation of detector performance. Low power-consuming and compact vacuum UV photosensors that can operate in direct contact with the scintillation gas, as an alternative to photomultiplier tubes, are now available. Small gettering devices with a low-power built-in heating elements have been shown to be sufficient for the required gas purification in GPSCs assembled with simple and inexpensive techniques, such as the use of epoxies for ceramic-to-metal joints. Copyright (c) 2001 John Wiley \& Sons, Ltd.
\end{abstract}

\section{INTRODUCTION}

Since their introduction, ${ }^{1}$ gas proportional scintillation counters (GPSCs) have been used for x-ray spectrometry in applications such as $\mathrm{x}$-ray astronomy, medical instrumentation and high-energy physics. ${ }^{2-8}$ In particular, they combine room temperature operation with relatively good energy resolution, large detection areas and high counting rates with reduced space charge effects, when compared with conventional gas proportional counters (GPCs).

The preferred absorption medium is a noble gas, usually xenon, chosen for its large ionization cross-section and high scintillation efficiency. A quartz-window photomultiplier tube (PMT) is usually used to detect the vacuum ultraviolet (VUV) electroluminescence produced in the scintillation region. However, the PMT not only limits the uniformity of the detection area of GPSCs but also increases their cost, power consumption, complexity, fragility and bulkiness. On the other hand, the necessity to use multi-electrode focusing structures in large-area GPSCs also contributes to the complexity and bulkiness of such detectors. Additionally, the requirement for high gas purity necessitates vacuum baked detectors with ceramic-to-metal joints and special gettering devices. These constraints are the main reasons for the almost exclusive use of GPSCs in very specific applications where detector cost, bulkiness and complexity are not limitations. These drawbacks are seldom a problem in applications to

*Correspondence to: J. M. F. dos Santos, GIAN-Departamento de Física, Universidade de Coimbra, Rua Larga, P-3004-516 Coimbra, Portugal. E-mail: jmf@gian.fis.uc.pt

Contract/grant sponsor: Fundação para a Ciência e a

Tecnologia (FCT); Contract/grant number: POCTI/43527/99.

${ }^{\dagger}$ Presented at the European Conference on EDXRS, Krakow,

Poland, 18-23 June 2000 areas such as energy-dispersive x-ray fluorescence (EDXRF) analysis and Mössbauer spectroscopy, but GPSCs remain broadly unknown and unused by the scientific and industrial community.

To the best of our knowledge only one of these detectors has been developed for application to EDXRF analysis and x-ray structure analysis and is produced commercially. ${ }^{9}$ The design contains only one focusing electrode so as to simplify substantially the manufacturing and, as a result, its performance is inferior to that of multi-electrode focusing designs. The detector window diameter is limited to $20 \mathrm{~mm}$ and the energy resolution degrades from 8.3 to $9.2 \%$ at $6 \mathrm{keV}$ as the window diameter is increased beyond $20 \mathrm{~mm}$.

In this paper, we review the state-of-the-art of GPSC technology and present research work developed in the University of Coimbra during the last decade. Recent advances towards the portability of simple, inexpensive and compact GPSCs are presented and discussed. These developments include alternatives to multi-electrode focusing structures, VUV photosensor alternatives to PMTs, gas purity and gettering, which are assessed for application of GPSC to EDXRF analysis. Future improvements in GPSC technology are discussed.

\section{OPERATION PRINCIPLE}

There are two types of x-ray gaseous detectors: ${ }^{10}$ the gas proportional scintillation counter (GPSC) and the gas proportional ionization avalanche counter [including proportional counters (PCs), multiwire gas chambers (MWGCs) and microstrip gas chambers (MSGCs)]. The x-ray absorption and primary electron drift processes are basically the same for both types of detectors: incident $\mathrm{x}$-rays interact 
Proportional counter

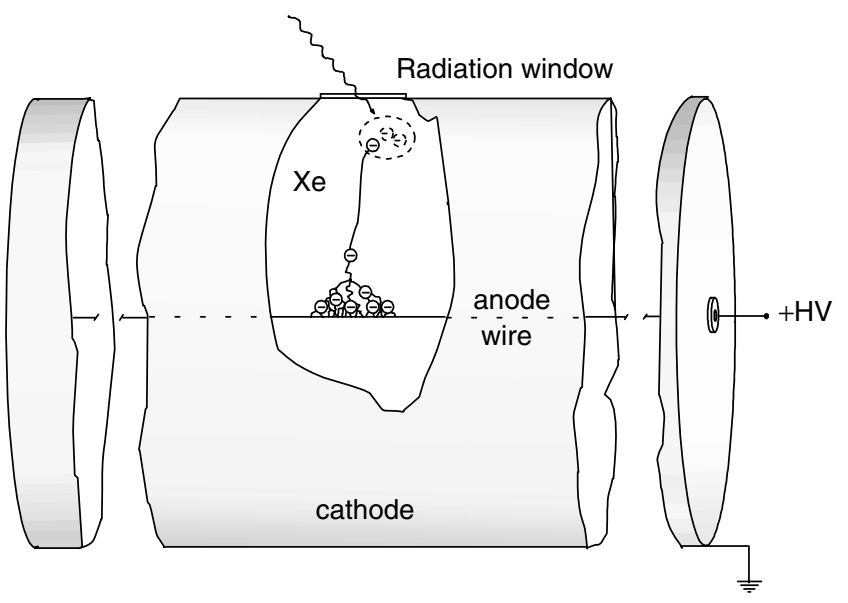

Gas proportional scintillation counter

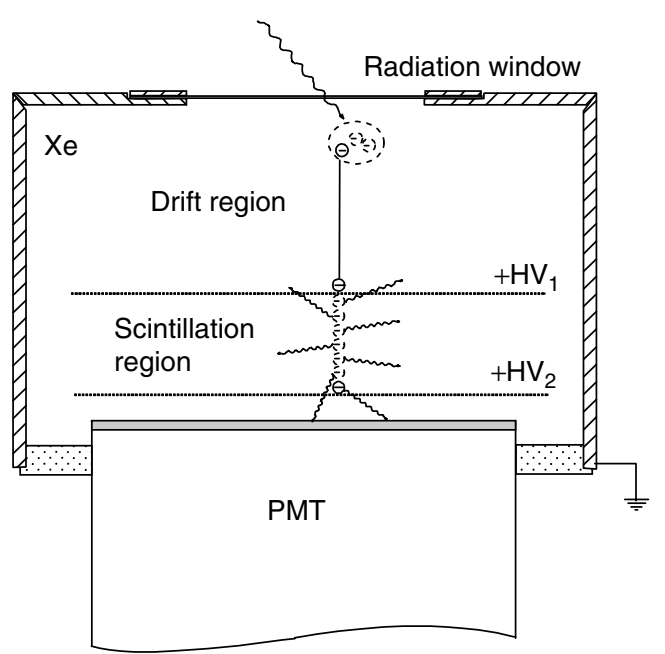

Figure 1. Schematic representation of the amplification processes in PCs and GPSCs.

primarily in a region where the electric field is weak-the drift region-and the resulting primary electron cloud drifts into a region with a stronger electric field (Fig. 1).

In proportional ionization counters, each primary electron produces an electron avalanche in the strong electric field near the anode, and a large number of secondary electrons are produced per primary electron in this amplification stage. In GPSCs the strong electric field is such that primary electrons excite but do not ionize the gas atoms, producing a light pulse as a result of the gas atom de-excitation, the socalled secondary scintillation or electroluminescence. In this way, in the amplification stage, a large number of photons are produced by each primary electron, and the signal amplification is achieved without space-charge accumulation, as in PCs.

Whereas in proportional avalanche counters the secondary electrons are directly collected in the anode, in GPSCs the scintillation photons are collected, instead, by a suitable photosensor. For both types of detectors the resulting pulses are proportional to the number of primary electrons produced in the drift region, and hence to the energy of the absorbed x-ray photon.

Proportional counters (PCs) that depend on the avalanche process for signal gain have achieved portability on the basis of their ruggedness, compactness and simplicity of operation, but at the expense of energy resolution. With the development of the enabling technologies that will be described in this review, we will show that the GPSC can be made equally compact, rugged and simple to fabricate and operate, with the advantage of a relatively good energy resolution.

The energy resolution, $R$, of a PC, is limited by the statistical fluctuations in the primary ionization, and also in the electron multiplication processes, and is given by ${ }^{10}$

$$
R=2.35 \sqrt{\frac{F}{\bar{N}}+\frac{1}{\bar{N}}\left(\frac{\sigma_{\mathrm{q}}}{G_{\mathrm{q}}}\right)^{2}}=2.35 \sqrt{\frac{F}{\bar{N}}+\frac{f}{\bar{N}}}
$$

where $\bar{N}$ is the average number of primary electrons produced per incident $x$-ray photon, $F=\left(\sigma_{N}^{2} / \bar{N}\right)$ is the relative variance of $\bar{N}$ (the Fano factor) and $f=\left(\sigma_{\mathrm{q}} / G_{\mathrm{q}}\right)^{2}$ is a measure of the fluctuations in the electron multiplication gain $G_{\mathrm{q}}$. For large gains, $f$ is independent of $G_{\mathrm{q}}$ and is larger than $F$ (e.g. $f=0.6$ and $F=0.2$ for xenon ${ }^{10,11}$ ). Thereby, the energy resolution of a PC is dominated by $f$.

On the other hand, the energy resolution, $R$, of a conventional GPSC is determined by the statistical fluctuations occurring in the primary ionization processes, in the production of VUV scintillation photons and in the photosensor, and can be given by ${ }^{12,13}$

$$
R=2.35 \sqrt{\frac{F}{\bar{N}}+\frac{1}{\bar{N}}\left(\frac{J}{\bar{N}_{\mathrm{s}}}\right)+\frac{1}{\bar{N}_{\mathrm{e}}}\left(1+\left(\frac{\sigma_{\mathrm{q}}}{G_{\mathrm{q}}}\right)^{2}\right)}
$$

where $\bar{N}_{\mathrm{s}}$ is the average number of scintillation photons produced per primary electron, $J$ is the relative variance of $\bar{N}_{\mathrm{s}}, \bar{N}_{\mathrm{e}}$ is the average number of photoelectrons produced in the photosensor per incident $\mathrm{x}$-ray photon absorbed in the drift region and $\left(\sigma_{\mathrm{q}} / G_{\mathrm{q}}\right)^{2}$ is a measure of the fluctuations in the photoelectron multiplication gain, $G_{\mathrm{q}}$, in the photosensor. The other parameters are defined as in Eqn (1). The second term of Eqn (2) can be neglected since $J \ll F^{13}$ and usually $N_{\mathrm{s}}>100$. Additionally, $\left(\sigma_{\mathrm{q}} / G_{\mathrm{q}}\right)^{2} \approx 1$ for a PMT photosensor. ${ }^{12}$ Thus, the energy resolution of a GPSC can be approximated by

$$
R=2.35 \sqrt{\frac{F}{\bar{N}}+\frac{2}{\bar{N}_{\mathrm{e}}}}
$$

In comparison with PCs, a very low additional uncertainty is introduced by the characteristic amplification processes in the GPSCs and the energy resolution is dominated by the statistical fluctuations in $\bar{N}$. Taking into account that $\bar{N}=E_{\mathrm{x}} / w$ (the x-ray photon energy, $E_{\mathrm{x}}$, divided by the mean energy to produce a primary electron, $w$ ) and defining the number of photoelectrons produced per primary electron, $L=\bar{N}_{\mathrm{e}} / \bar{N}^{14}$ the energy resolution can be given by

$$
R=2.35 \sqrt{\frac{w}{E_{\mathrm{x}}}\left(F+\frac{2}{L}\right)}
$$


Both $w$ and $F$ are characteristics of the gas but they depend on the x-ray energy. ${ }^{15} L$ is a parameter that describes the photosensor performance. Energy resolutions as low as 7.6\% have been obtained for xenon-filled GPSCs, corresponding to $L$ values above 20 . The best performance achieved with xenon-filled PCs is $16 \%$.

GPSCs can be successfully fabricated with different geometries. In single-electrode detectors, having a wire ${ }^{1,16}$ or a spherical anode, ${ }^{17}$ the electric field increases continuously, the scintillation region being located around the anode. In the uniform field geometry ${ }^{18}$ and in the spherical field geometry, ${ }^{5}$ the scintillation region is limited by two grid electrodes, the drift region being limited by the detector radiation entrance window and the first grid (Fig. 1). For the latter cases, it is convenient for the scintillation-todrift electric fields ratio to be higher than 5, in order to guarantee full transmission of primary electrons through the first grid. ${ }^{19}$ The uniform field geometry yields higher scintillation efficiency than the spherical and the wire anode geometries. $^{20}$

A driftless GPSC design, a GPSC without drift region where the $x$-rays are directly absorbed in the scintillation region, has also been introduced. ${ }^{6,12}$ Owing to the high electric field in the scintillation region, the degrading influence of primary electron losses to the detector radiation window ${ }^{21}$ is less than in conventional GPSCs. Several other effects, such as the lateral diffusion of the primary electron cloud and primary electron losses to impurities and to the low-voltage grid, are also minimized. However, in a driftless GPSC the observed light amplitude is dependent on the distance covered by the primary electron cloud in the scintillation region, and thus on the absorption depth of the incident $x$-ray photon. The scintillation-burst time duration is correlated with this distance and allows pulseamplitude correction, restoring the proportionality between the corrected pulse amplitude and the corresponding $x-$ ray photon energy. For low-energy x-rays with absorption lengths much smaller than the scintillation region thickness, pulse time duration analysis is therefore not necessary.

\section{SECONDARY SCINTILLATION PRODUCTION}

The secondary scintillation mechanisms have been studied in detail in earlier publications. ${ }^{22-24}$ For gas pressures above a few tens of $\mathrm{kPa}$, the electroluminescence of noble gases presents a narrow continuum, peaking at $171 \mathrm{~nm}(7.3 \mathrm{eV})$ for xenon, at $148 \mathrm{~nm}(8.4 \mathrm{eV})$ for krypton and at $128 \mathrm{~nm}(9.8 \mathrm{eV})$ for argon with full widths at half-maxima of 12,10 and $10 \mathrm{~nm}$ $(\sim 1 \mathrm{eV})$, respectively.

The electroluminescence yield, $Y$, the average number of scintillation photons produced per primary electron and per unit length, has been studied in detail. Experimental results obtained by different workers have been compiled. ${ }^{25}$ Santos et al. ${ }^{26}$ carried out detailed Monte Carlo (MC) simulation studies of the electron drift in xenon under the influence of an electric field and of the production of electroluminescence.

In Fig. 2 we present Monte Carlo simulation results of the reduced electroluminescence yield, $Y / p$ (the electroluminescence yield, $Y$, divided by the pressure, $p$ ), as a function of

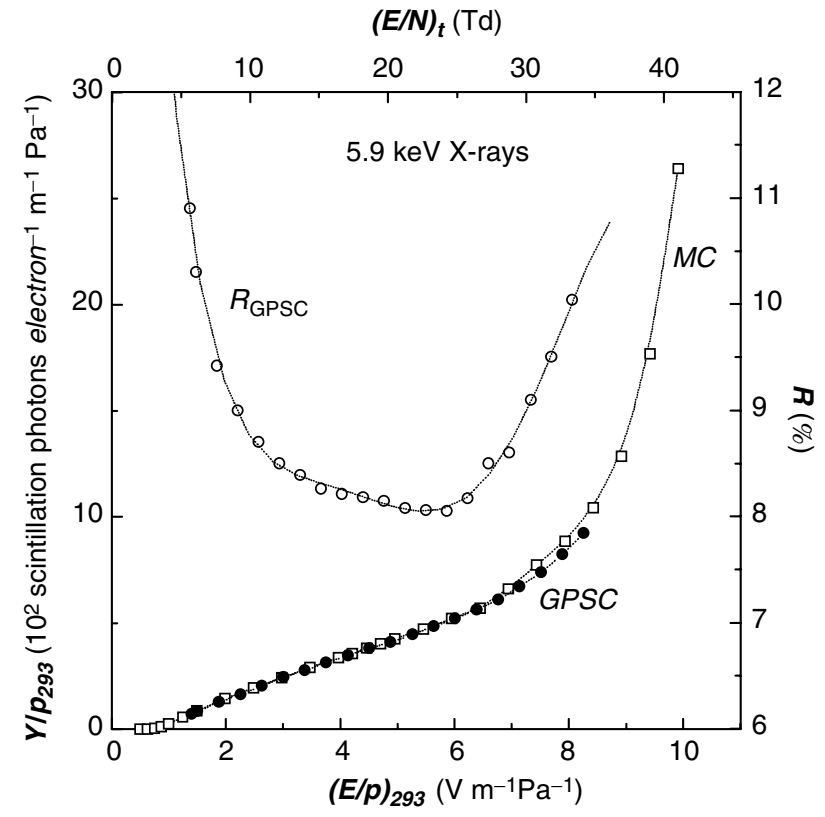

Figure 2. Reduced scintillation yield ( $\square$, Monte Carlo Results; -, experimental results) and detector energy resolution $(0)$ as a function of the reduced electric field in the scintillation region for $5.9 \mathrm{keV} \mathrm{x}$-rays. The experimental values have been normalized to the Monte Carlo value at $E / p=4.5 \mathrm{~V} \mathrm{~m}^{-1} \mathrm{~Pa}^{-1}$.

the reduced electric field, $E / p$, in the scintillation region for $5.9 \mathrm{keV} x$-rays, in xenon. Figure 2 also presents experimental results for the average pulse amplitude and detector energy resolution as a function of the reduced electric field in the scintillation region for a $1 \mathrm{~mm}$ collimated $5.9 \mathrm{keV}$ x-ray beam, obtained with a uniform electric field pure-xenon GPSC. ${ }^{27}$ The experimental values have been normalized to the calculated $\mathrm{MC}$ value at $E / p=4.5 \mathrm{~V} \mathrm{~m}^{-1} \mathrm{~Pa}^{-1}$. As can be seen, above a scintillation threshold at about $0.75 \mathrm{~V} \mathrm{~m}^{-1} \mathrm{~Pa}^{-1}$ the scintillation yield follows an approximately linear trend until an exponential increase due to charge multiplication becomes evident. The best energy resolution is obtained for $E / p$ values above the xenon ionization threshold $\left(\sim 4.5 \mathrm{~V} \mathrm{~m}^{-1} \mathrm{~Pa}^{-1}\right.$ for $x^{x e n o n}{ }^{27,28}$ ) before the contribution of charge multiplication fluctuations ( $f$ parameter) becomes significant. The onset for the energy resolution degradation is also determined by the $L$ value. Low $L$ values will tolerate higher degrees of electron multiplication (e.g. see Ref. 29); although the first term under the square root sign in Eqn (3) increases owing to the presence of $f$, as in Eqn (1), this is compensated for by a reduction in the second term as $\bar{N}_{\mathrm{e}}$ increases when secondary electrons are produced in the scintillation region.

The analysis of Fig. 2 shows that, for pure xenon GPSCs, the reduced electric field in the drift region must be lower than $0.75 \mathrm{~V} \mathrm{~m}^{-1} \mathrm{~Pa}^{-1}$, the xenon scintillation threshold, and the reduced electric field in the scintillation region should not exceed the onset for the degradation of energy resolution. For the drift region, reduced electric fields as low as 0.08 or $0.15 \mathrm{~V} \mathrm{~m}^{-1} \mathrm{~Pa}^{-1}$ can be used. However, for soft $\mathrm{x}$-rays whose interactions occur mainly near the detector radiation window, drift electric fields as high as possible are advised, owing to the backscattering and the loss of primary electrons to the radiation entrance window, resulting in a degradation 
of the detector response. ${ }^{21}$ The pulse-height spectra exhibit distortions represented by a significant departure from the Gaussian shape output due to a tail that extends down to very low amplitudes.

\section{GPSC PERFORMANCE CHARACTERISTICS}

The characteristics of GPSCs in x-ray spectrometry allow their use in the energy range from 0.1 to $100 \mathrm{keV}, 6,12,30,31$ with an efficiency depending on the drift region depth and on the gas pressure. Among room temperature detectors, GPSCs present the best energy resolution for large detection areas. Detection areas up to several hundred $\mathrm{cm}^{2}$ have been described. ${ }^{5,32}$ For x-ray energies below $1-2 \mathrm{keV}$, GPSC energy resolutions can even be better than those obtained for cooled solid-state detectors [HPGe and $\mathrm{Si}(\mathrm{Li})$ ], when sensitive areas of several $\mathrm{cm}^{2}$ are required. Energy resolutions as low as 30, 14, 7.6, 4.3 and $3.3 \%$ have been reported for $0.3,1.5,6,22$ and $60 \mathrm{keV}$, respectively. ${ }^{2,18,31-34}$ The GPSC energy resolution follows a linear dependence on $E_{\mathrm{x}}^{-1 / 2}$, but a gradual deviation from this trend is observed for high-energy x-rays, depending on the relative number of x-ray interactions in the scintillation region and on the dimensions of the primary electron cloud reaching the scintillation region, when compared with the photosensor dimensions.

The energy linearity of gaseous detectors is very good except for the regions around the fill gas atoms' absorption edges. Deviations from linearity in the response of gaseous detectors are well documented, and a review was presented by Dias et al. ${ }^{15}$ in which a quantitative explanation was supported by MC simulation. It was shown that the departure from linearity of the detector energy response occurs at the fill gas absorption edges, owing to differences in the energy expended by the initially photoionized atom in establishing the ground state, as different shells are excited. If a new photoionization channel becomes energetically accessible, the photoelectron energy becomes smaller and the subsequent de-excitation cascade of the photoionized atom results in a higher number of electron vacancies in the outermost shells. A measurable amount of the absorbed energy can be expended in establishing the ground state of the excited ion. At still higher energies, the energy dissipated in establishing the cascade vacancies is a smaller fraction of the total energy transferred to photoelectrons, and approximate energy linearity is restored. This effect is not noticeable in solid-state detectors $\left[\mathrm{HPGe}^{35}\right.$ and $\mathrm{Si}(\mathrm{Li})^{36}$ ] owing to collective effects present in the crystalline structure
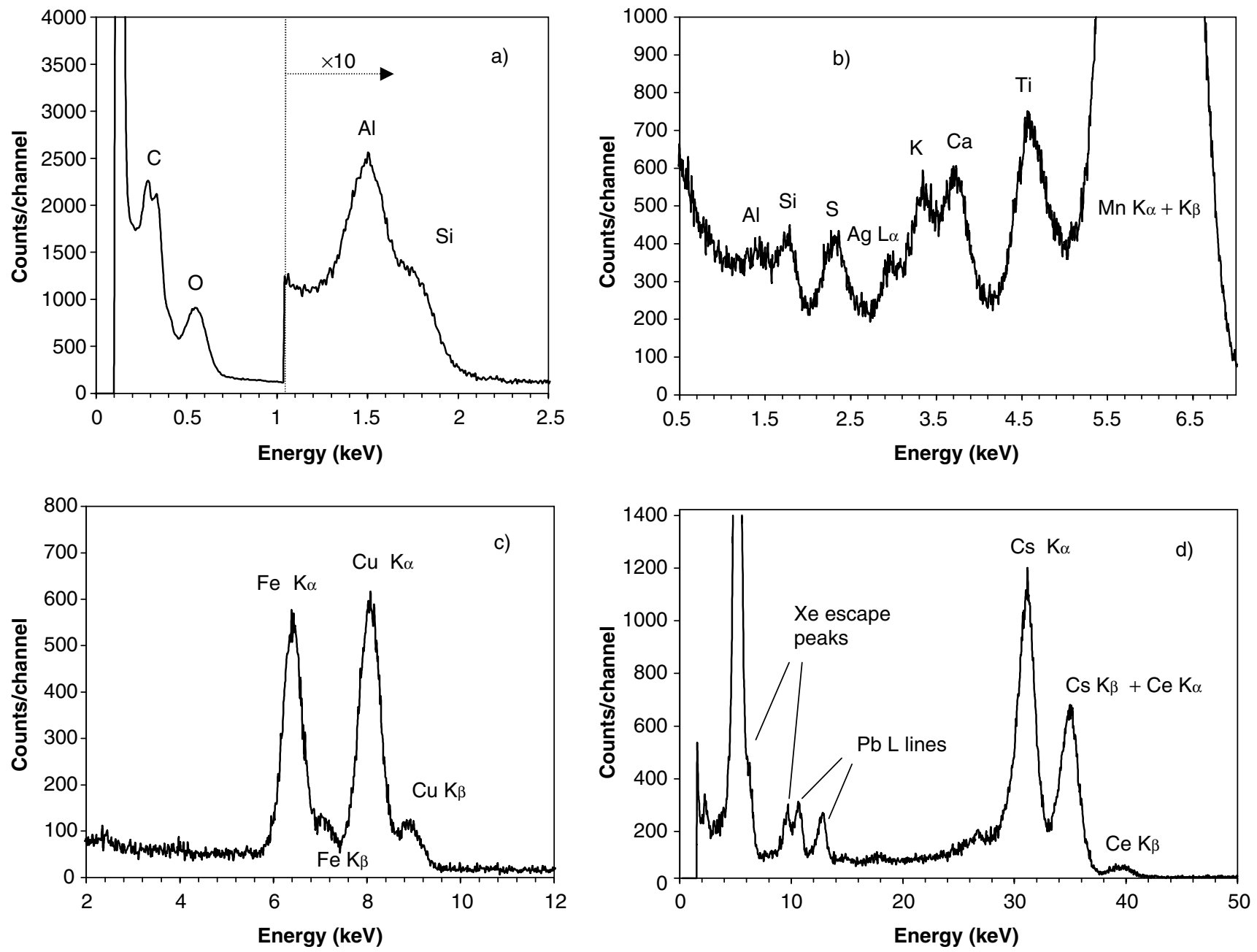

Figure 3. Typical x-ray fluorescence spectra of some samples: (a) andalusite; (b) anthracite; (c) non-homogeneous pyrite/calcopyrite; (d) cerium and caesium oxide mixture. 
where the photoionized atom is located. The response of a xenon gas detector to $\mathrm{x}$-rays presents a sudden decrease of about $0.6,1.3,0.4$ and $0.2 \%$ in the average number of primary electrons at the xenon $\mathrm{K}(34.564 \mathrm{keV}), \mathrm{L}_{3}(4.782 \mathrm{keV}), \mathrm{L}_{2}$ (5.104 keV) and $\mathrm{L}_{1}(5.453 \mathrm{keV})$ edges, respectively.

Some examples of XRF spectra from different samples are presented in Fig. 3. All these examples were obtained with different conventional, non-focused, uniform-field type GPSCs of simple design. ${ }^{18}$ Figure 3(a) depicts the XRF spectrum of a homogeneous andalusite sample $(56.43 \%$ $\mathrm{AlO}_{2}, 40.99 \% \mathrm{SiO}_{2}$ ), measured in vacuum and excited with $\alpha$-particles, obtained with a GPSC using a special entrance window with high transparency to soft $x$-rays. ${ }^{37}$ The oxygen line can clearly be distinguished, with an energy resolution of about $20 \%$. The nitrogen and carbon lines of the window material can also be seen above the electronic noise. Figure $3(\mathrm{~b})$ presents the $\mathrm{x}$-ray spectrum of an anthracite sample $\left(0.23 \% \mathrm{Na}_{2} \mathrm{O}, 0.11 \% \mathrm{MgO}, 1.27 \% \mathrm{AlO}_{2}, 2.47 \% \mathrm{SiO}_{2}\right.$, $0.58 \%$ total $\mathrm{S}, 0.11 \% \mathrm{~K}_{2} \mathrm{O}, 0.11 \% \mathrm{CaO}, 0.06 \% \mathrm{TiO}_{2}, 0.25 \%$ $\mathrm{Fe}_{2} \mathrm{O}_{3}$ ), measured in air and excited with a ${ }^{55} \mathrm{Fe}$ x-ray source, obtained with a detector using a $7.5 \mu \mathrm{m}$ Kapton entrance window, with the sample placed $1 \mathrm{~cm}$ from the detector window. All the different element lines are distinguished. In particular, the $\mathrm{S}$ and Ti lines are completely separated from the others, allowing simpler and more accurate analysis than with PCs. The energy resolutions obtained were 11.8 and $8.5 \%$ for the $\mathrm{S}$ and $\mathrm{Ti}$ lines, respectively. Figure 3(c) depicts the XRF spectrum of a non-homogeneous calcopyrite sample excited with a ${ }^{109} \mathrm{Cd}$ x-ray source. The Fe and $\mathrm{Cu}$ $\mathrm{K} \alpha$ and $\mathrm{K} \beta$ lines are partially separated and the $\mathrm{K} \alpha$ lines of those elements are completely separated, a performance that cannot be achieved with PCs. In Fig. 3(d), the XRF spectrum of a non-homogeneous sample of Cs and Ce oxides, excited with a ${ }^{241} \mathrm{Am} \gamma$-ray source, is presented. The spectral features include the $\mathrm{Cs}$ and $\mathrm{Ce} \mathrm{K} \alpha$ and $\mathrm{K} \beta$ lines, the lead collimator $\mathrm{L}$ lines and the xenon escape peaks. For the detector used, ${ }^{38}$ energy resolutions of $5.5 \%$ were obtained for the Cs and Ce $\mathrm{K} \alpha$ and $\mathrm{K} \beta$ lines.

It is apparent from the data presented here that the improved energy resolution inherent in the scintillation process enables the GPSC to distinguish XRF features that are not present with an avalanche proportional counter. Most significantly, the greater detail in the pulse-height distributions as a result of the improved GPSC energy resolution allows the quantification of complex spectra with relatively straightforward deconvolution algorithms that can be included in a portable device. Even when compared with the anticipated performance of the next generation of room temperature solid-state detectors, the portable GPSC will have advantages in situations where large detector sensitive areas are required.

\section{ADVANCES TOWARDS PORTABILITY}

\section{Alternatives to multi-electrode focusing}

The high performance of GPSCs degrades when the detection areas are large compared with the photosensor area. This is due to the dependence of the number of scintillation photons collected by the photosensor at the x-ray interaction position. This effect results from the variation of the solid angle subtended by the photosensor relative to the region where the scintillation occurs, ${ }^{39-41}$ and is the most important effect for the energy resolution degradation of GPSCs using large detection areas.

To overcome this effect, multi-electrode focusing lenses have been introduced in the GPSC drift region, in order to confine in a small volume the region where the scintillation occurs, thereby reducing the variation of the solid angle subtended by the photosensor. ${ }^{5,30,42}$ With these techniques, the detector becomes more bulky, complex and expensive, a drawback for portable devices. Recent work has been carried out on the development of simpler and more cost-effective
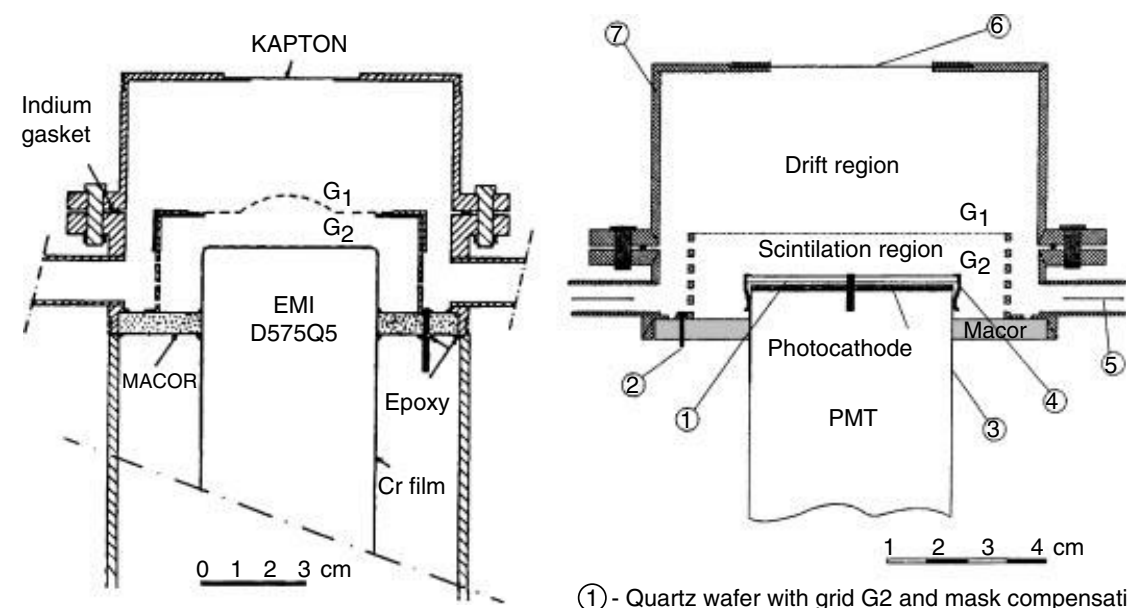

(1) - Quartz wafer with grid G2 and mask compensation

(2) - G1 feedthrough

(3) - Chromium film

(4) - Electrical contacts for G2

(5) - To gas purifier

(6) - Kapton window, $75 \mu \mathrm{m}$ thick

(7)- Stainless steel

a)

Figure 4. Schematic diagram of (a) the curved-grid GPSC and (b) the masked-photosensor GPSC. 
GPSC $^{18,31,43,44}$ for EDXRF applications, where they could be a good alternative to conventional proportional counters.

It has been shown that the best design performance for a GPSC is obtained by placing the photosensor closer to the scintillation region. The advantages of this design lead not only to an improvement in light collection but also, and more important, to a small dependence on the solid angle effect. The commonly used 1-2 cm distances are the worst-case situation in terms of this dependence. ${ }^{41} \mathrm{On}$ the other hand, the relative importance of the photosensor spatial uniformity on the GPSC performance depends on the photosensor-to-scintillation region distance and on the detector design. It has been shown that the commonly used venetian blind PMT could limit the performance of GPSCs owing to its poor uniformity. Consequently, the linear focused PMT type is advisable for improved detector performance. ${ }^{45}$ Implementing the two measures described above, energy resolutions of $7.9,8.7$ and $9.3 \%$ were obtained for a $5.9 \mathrm{keV}$ and for a 2, 20 and $25 \mathrm{~mm}$ collimated x-ray beam, respectively, using a non-focused uniform-field GPSC equipped with a 2 in PMT. ${ }^{18}$

As an alternative to multi-electrode focusing techniques, the use of curved-grid and masked-photosensor techniques ${ }^{31,43,44}$ allow the construction of non-focused GPSCs with good energy resolution while maintaining a medium/large detection area with relatively small photomultiplier tubes (Fig. 4). The curved-grid technique uses a first curved grid and a second planar grid, instead of two parallel planar grids, for the confinement of the scintillation region. The electric field intensity increases radially (relative to the PMT axis), hence the electroluminescence increases with the radial position of its production, in such a way that this radial increase compensates for the radial decrease of the solid angle subtended by the PMT. The mask technique uses two parallel planar grids to confine the scintillation region but the photosensor is now covered with a mask with a light transmission that increases radially in such a way that compensates for the radial decrease in the solid angle. Thus, the amount of scintillation light reaching the photosensor will be constant and independent of the position where the scintillation occurs. For a given geometry, the shape of the curved grid or the mask has to be calculated.

These techniques allowed the use of detector radiation windows with dimensions similar to those of the PMTs, up to $80 \%$ of the PMT diameter, ${ }^{31,43}$ without degradation of the detector energy resolution, and result in designs that are structurally simpler and less expensive than those with focusing. For $5.9 \mathrm{keV}$ x-rays energy resolutions of $8.0 \%$ are obtained with a curved grid GPSC, having a $25 \mathrm{~mm}$ diameter radiation window and being equipped with a 2 in PMT. ${ }^{46}$

Another alternative to solid angle compensation can be implemented using a position-sensitive photosensor. ${ }^{34}$ The knowledge of the radial position of the $x$-ray interaction allows for solid angle compensation by means of real-time pulse amplitude correction, after the position determination.

\section{Photosensor alternatives to PMTs}

PMTs provide high gains with low noise and can present large active areas. However, they have limited spatial uniformity and they increase the size, cost, power consumption, complexity and fragility of the otherwise simple and robust GPSC, presenting a drawback in applications to areas such as portable devices for EDXRF analysis, Mössbauer spectroscopy and operation in strong magnetic fields. Additionally, they require high-purity VUV quartz windows, which partially absorb the scintillation light, especially the thick windows required for high-pressure applications.

Alternatives to replace the PMT by a more convenient photosensor have been investigated. These included multiwire proportional chambers ${ }^{47-49}$ with UV-sensitive filling gases [photoionization detectors (PIDs)], microstrip gas chambers (MSGCs), ${ }^{50}$ microchannel plates ${ }^{51}$ and standard photodiodes. ${ }^{52}$ Although PID photosensors and MSGCs can be implemented with large areas, they have fill gases different from those used in GPSCs, with inherent problems of handling, gas purity and ageing. On the other hand, photodiodes present an attractive alternative to PMTs as the GPSC photosensor. They are simple to operate, have very low power consumption and high quantum efficiency and can be operated in high-intensity magnetic fields. However, until recently, their sensitive range excluded the VUV region and they were operated outside the detector together with an organic wavelength shifter placed on the inside of the exit scintillation window, a serious drawback for gas purity and long-term operation stability. Both photodiodes and microchannel plates have limited area sizes.

Typically, all these photosensors were independent devices coupled to the GPSC by means of an intervening VUV window. However, integrated photosensors that can be placed inside the GPSC envelope, in direct contact with the gas, are of great interest. They avoid the use of an interface VUV window that can be expensive, difficult to handle and absorb some of the scintillation light.

The use of a CsI-covered microstrip plate (MSP) as an integrated photosensor replacing the PMT in a GPSC was investigated, ${ }^{29}$ and is depicted in Fig. 5. It was shown that a CsI-covered MSP is an attractive alternative to the PMT as an integrated photosensor for GPSCs in applications where cost, compactness, large area and power consumption are important criteria, with the possibility of working at higher pressures than standard GPSCs. However, detector energy resolutions of 12,7 and $5.5 \%$ obtained for 5.9, 22.1 and $59.6 \mathrm{keV}$, respectively, are worse than those achieved with a PMT-based GPSC, although better than those achievable with proportional counters or microstrip gas chambers. A poor $L$ value is achieved with this photosensor [Eqn (4)]. Further studies to improve the performance of this integrated photosensor are being carried out, namely the optimization of the MSP design ${ }^{29}$ and the development of different type of microstructures ${ }^{53}$ to improve the $L$ value.

Recently, large-area avalanche photodiodes (LAAPDs) have been developed, delivering high gains with improved spatial uniformity. Windowless LAAPDs with extended sensitivity to the VUV region are now commercially available ${ }^{54}$ (Advanced Photonix, Camarillo, CA; Radiation Monitoring Devices, Watertown, MA, USA). These photodiodes can operate within the gas envelope, without the requirement for a detector scintillation window that has less than full 


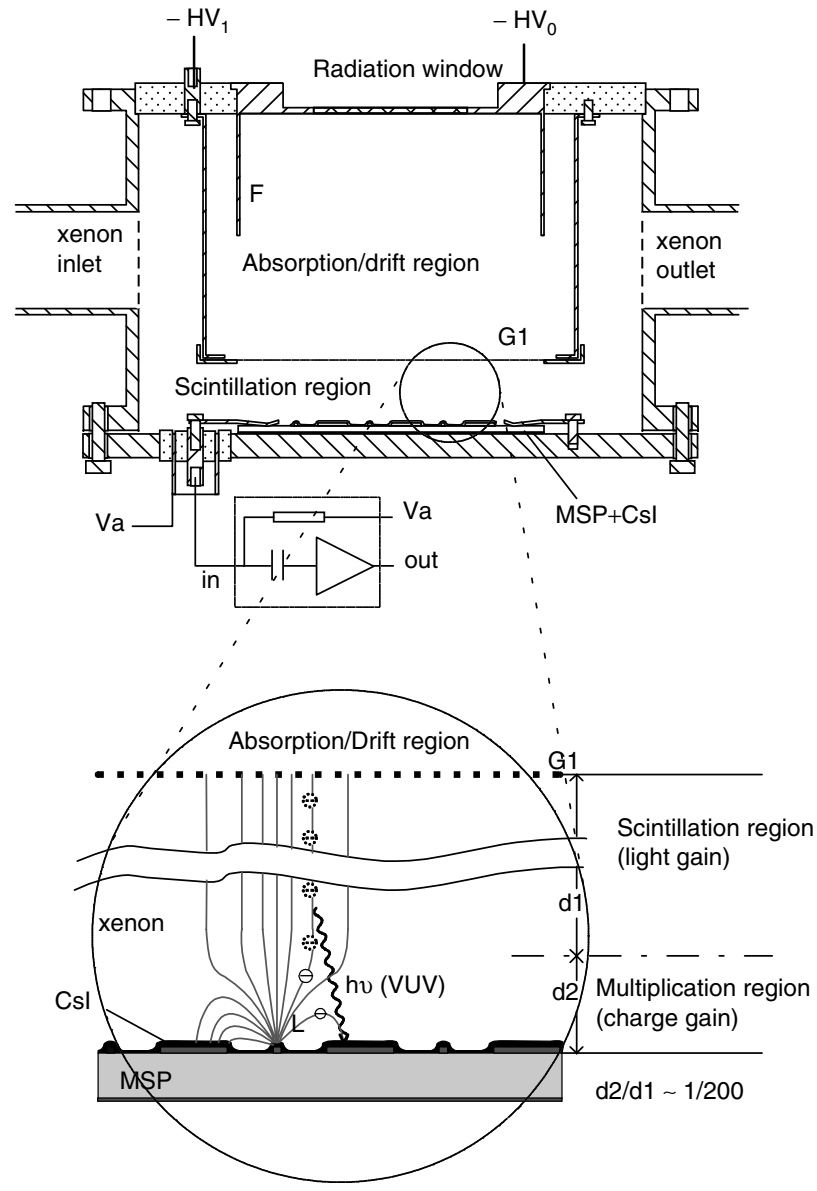

Figure 5. Schematic diagram of a GPSC instrument with a Csl-covered MSP.

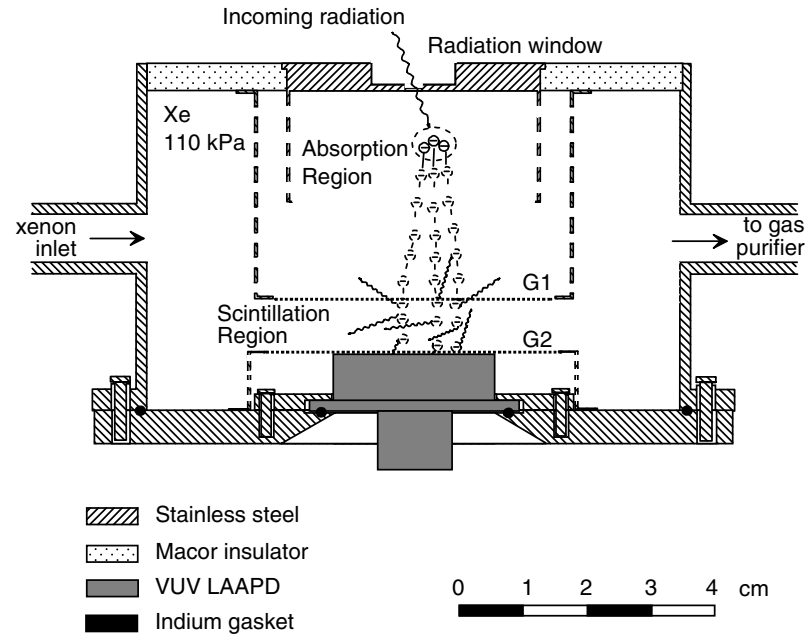

Figure 6. Schematic diagram of a GPSC instrument with an LAAPD.

transmission of the VUV light. The use of a $16 \mathrm{~mm}$ diameter VUV LAAPD as the photosensor in a xenon-filled GPSC has been investigated, ${ }^{55}$ as shown in Fig. 6. Energy resolutions of 7.8 and $4.4 \%$ were obtained with a xenon-filled GPSC for 5.9 and $22.1 \mathrm{keV}$ x-rays, respectively. This performance is better than that achieved with GPSCs of similar design, equipped with small-area $38 \mathrm{~mm}$ PMTs, ${ }^{9,43}$ or even with $51 \mathrm{~mm}$ PMTs. ${ }^{18,28,38}$
A further alternative under study is the use of largearea conventional VUV photodiodes. ${ }^{56}$ Since these conventional diodes do not need a biasing voltage for optimum operation, ${ }^{56}$ they can be an attractive alternative photosensor readout.

The present results ${ }^{29,55}$ demonstrate that integrated photosensors, as an alternative to PMTs, are feasible. Although the performance obtained with the CsI-covered MSP photosensor is worse than that obtained with the LAAPD, the large-area capability of the former may be an important advantage in specific applications, e.g. the x-ray spectroscopy of muonic hydrogen for the Lamb shift measurement experiment. ${ }^{57}$ The largest commercially available LAAPD measures $16 \mathrm{~mm}$ in diameter. Both solutions result in simple, compact, low power consumption and robust detectors, a significant advance towards the feasibility of portable highperformance x-ray spectrometry systems based on GPSC technology.

\section{Gas purity and gettering}

Since the early work, it was found that gas purity is an important factor for obtaining a good performance of GPSCs. The requirement for high gas purity led to the necessity for vacuum-baked detectors, assembled with ceramic-to-metal joints and using ultra-high-vacuum (UHV) techniques. These techniques allow the production of sealed detectors with lifetimes of several years. ${ }^{9}$ However, these same techniques increase the complexity and cost of GPSCs production. Most of the GPSCs described in the literature were either sealed detectors coupled to an appendage getter pump, or detectors coupled to a gas purification system with gas circulation through getters.

Monte Carlo simulation studies of the electroluminescence production in the GPSC scintillation region ${ }^{26}$ have shown that the number of elastic collisions experienced by each primary electron, before it can gain from the electric field enough energy to excite the gas atoms, is very large $\left(\sim 10^{4}\right)$. The collision with an impurity molecule may result in some loss of the electron kinetic energy through rotational and vibrational relaxation, without radiative emission, resulting in a reduction of the electroluminescence yield. ${ }^{23}$ If the probability of electron collision with an impurity molecule is significant, the gas scintillation efficiency is reduced and the detector performance degrades. The primary electron loss to electronegative impurity atoms may also be important during the drift process in the weak electric fields of the drift region. ${ }^{58}$

Low vapour pressure and low activation and operation temperatures are three important characteristics of the getter material. A Zr-V-Fe alloy (SAES St $707^{59}$ ) has been used for this purpose. The St 707 activation temperature can be as low as $350^{\circ} \mathrm{C}$ and getter steady operation temperatures of $100-150^{\circ} \mathrm{C}$ have proved to be sufficient for efficient gas purification. Getter heating is done by temperaturecontrolled electric heating tapes from the outside of the getter tube container.

Our research work at Coimbra has shown that GPSCs assembled without the use of UHV techniques, and using epoxies instead of ceramic-to-metal welding, can achieve 
comparable detector performances to the others. Such a GPSC type $^{44}$ [Fig. 4(b)], where the gas circulates through convection in a closed circuit around the detector (the getter tube has about 50 pieces of St 707/washer/833), is still operating after more than 7 years, without a noticeable performance degradation. It was also found that a small quantity of getter is efficient enough for maintaining gas purity. A small getter unit (St 172/HI/7-6/150C), with a built-in resistive heating element $(\sim 1 \Omega)$, has proved to be sufficient for maintaining gas purification in a $\sim 1 \mathrm{dm}^{3}$ volume detector ${ }^{31}$ for more than 6 months. Hence it is possible to have high-performance sealed GPSCs, assembled with epoxies, using a single getter element, which has a total power consumption of around $1 \mathrm{~W}$.

\section{Biasing and pulse analysis electronics}

Modular low-power and compact electronics for GPSCs are already being used., ${ }^{9,60}$ The GPSC electrode grids voltage biasing (typically 5-6 kV) that delimit the scintillation region do not require low ripple power supplies. However, as the GPSC signal is taken from the photosensor anode, a low ripple power supply (typically $1-2 \mathrm{kV}$ ) is required to polarize the photosensor, since this ripple is added to the signal. If the photosensor is placed near the scintillation region, to maximize the scintillation light collection, either the detector radiation entrance window is polarized at negative high voltage and the photosensor photocathode at ground potential, ${ }^{9}$ a drawback for laboratory and portable applications, or else the photosensor must be floating over the high voltage applied to the grids of the scintillation region. ${ }^{18,31,43,44}$ In this latter case both power supplies must have low ripple.

However, the recent progress achieved in optocoupling electronics provides an alternative solution to the ripplesensitive capacitive high-voltage coupling. If the output signal is taken from the floating anode with an optocoupled amplifier, the ripple of the high-voltage line will no longer be added to that signal and a small, low-current, high-voltage power supply with a ripple that can be as large as a few volts is sufficient for biasing the high-voltage grids. ${ }^{60}$ Only the floating photosensor high-voltage supply is required to have low ripple. The whole electronic system can then be of low power, compact and cost-effective, thus being applicable to portable GPSC-based systems.

Nowadays, pulse shaping and analysis can be efficiently performed using digital signal acquisition and processing techniques. These techniques have clear advantages over analogue methods, due not only to the powerful pulse manipulation, but also to the lower complexity of the electronic system. A simple digital pulse-height analyser (DPHA) can be used instead of the multichannel analyser (MCA), performing pulse integration, pulse timing analysis, baseline restoration and pulse pile-up correction, in addition to pulse discrimination for peak enhancement and background reduction. The study of the application of digital signal processing techniques to GPSCs is presented in Refs 61-64.

Figure 7 depicts an example of the pulse-height distributions obtained from lead ( $100 \mathrm{ppm})$ in a water sample excited with a ${ }^{109} \mathrm{Cd} \mathrm{x}$-ray source, taken with a Wilkinsontype ADC card MCA (curve a) and with a DPHA (curve b).

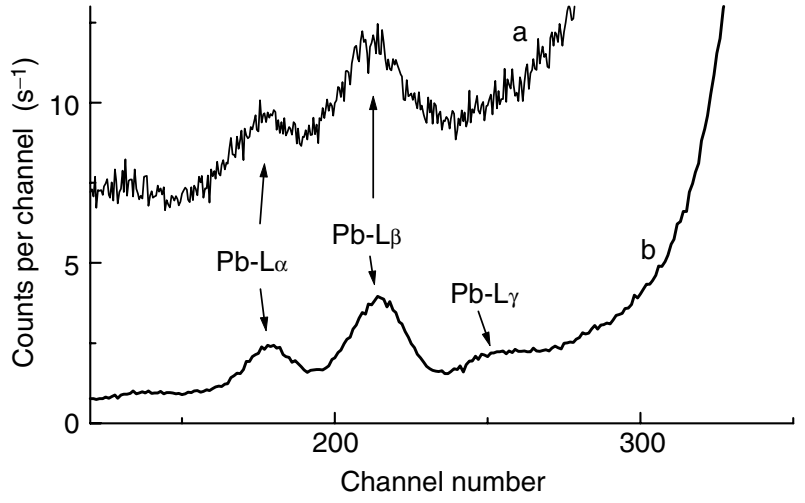

Figure 7. Pulse-height distributions obtained from lead $(\sim 100 \mathrm{ppm})$ in a water sample excited with a ${ }^{109} \mathrm{Cd}$ source for a total count rate of about $18000 \mathrm{~s}^{-1}$, obtained with (a) a standard Wilkinson-type PHA and (b) a DPHA.

An energy resolution and a peak-to-background ratio for the $\mathrm{Pb} \mathrm{L} \beta$ line $(12.6 \mathrm{keV})$ of $9.3 \%$ and 0.29 were obtained when using the Wilkinson ADCs MCA, whereas values of $7.9 \%$ and 1.7 were achieved when using the DPHA with pulse duration discrimination for counting peak efficiencies of more than $90 \%$.

\section{CONCLUSIONS}

Recent research on GPSCs has shown the feasibility of producing simple, compact and inexpensive sealed detectors for portable XRF systems. Low power-consuming VUV photosensors that can operate in direct contact with the scintillation gas are being investigated and have the capability of replacing PMTs with advantage. Small gettering devices with a low-power built-in heating element have been shown to be sufficient for the required gas purification in GPSCs assembled with simple and low-cost techniques, such as the use of epoxies for ceramic-to-metal joints. Compensation for solid angle effects using the curved-grid technique can produce non-focused GPSCs with medium-sized radiation entrance windows, at least up to $80 \%$ of the photosensor active diameter, without degradation of the detector performance. Modular biasing and pulse analysis electronics can be made compact and inexpensive for the relatively small power consumption needs of the overall spectrometer system.

\section{Acknowledgements}

The work was carried out in the Atomic and Nuclear Instrumentation Group of the Instrumentation Center (Unit217/94) of the Departamento de Física, Universidade de Coimbra. Support is acknowledged from the Fundação para a Ciência e a Tecnologia (FCT), project POCTI/43527/99. Thanks are due to C. M. B. Monteiro for revising the manuscript.

\section{REFERENCES}

1. Conde CAN, Policarpo AJPL. Nucl. Instrum. Methods 1967; 53: 7.

2. Policarpo AJPL. Space Sci. Instrum. 1977; 3: 77.

3. Ngoc HEN, Jeanjean J, Itoh H, Charpack G. Nucl. Instrum. Methods 1980; 172: 603.

4. Perez-Mendes LBLV. In Instrumentation in Elementary Particle Physics, Fabjian CW, Pilcher JE (eds). World Scientific: Singapore, $1987 ; 149$. 
5. Lamb P, Manzo G, Re S, Boella G, Villa G, Andresen R, Sims MR, Klark GF. Astrophys. Space Sci. 1987; 136: 369.

6. Smith A, Peacock A, Kowalski TZ. IEEE Trans. Nucl. Sci. 1987; 34: 57.

7. Smith A, Bavdaz M. Rev. Sci. Instrum. 1992; 63: 683.

8. Varvaritsa VP, Vikulov IV, Ivashov VV, Panov MA, Filatov VI, Schekin KI. Instrum Exp. Tech. 1992; 35: 745.

9. Goganov DA, Shultz AA. Nucl. Instrum. Methods A 1997; 394: 151.

10. Knoll GF. Radiation Detection and Measurements (3rd edn). Wiley: New York, 1999; chapt. 5 and 6.

11. Rachinhas PJBM, Dias THVT, Stauffer AD, Santos FP, Conde CAN. IEEE Trans. Nucl. Sci. 1996; 43: 239.

12. Simons DG, de Korte PAJ. Nucl. Instrum. Methods A 1989; 277: 642.

13. Dias THVT. PhD Thesis, Universidade de Coimbra, 1986.

14. Policarpo AJPL. Nucl. Instrum. Methods 1978; 153: 389.

15. Dias THVT, dos Santos JMF, Rachinhas PJBM, Santos FP, Conde CAN, Stauffer AD. J. Appl. Phys. 1997; 82: 2742.

16. Garg SP, Murthy KBS, Sharma RC. Nucl. Instrum. Methods A 1995; 357: 406.

17. Policarpo AJPL, Alves MAF, Leite MSSCP, Santos MCM. Nucl. Instrum. Methods 1974; 118: 221.

18. dos Santos JMF, Bento ACSS, Conde CAN. X-Ray Spectrom. 1993; 22: 328 .

19. Borges FIGM, Conde CAN. In Proceedings of SASP 94, 20-24 March, Hintermoos, Austria, Märk TD, Schrittwieser R, Smitt D (eds). Institute für Ionen Physik, Universität Innsbruck: Innsbruck, 1994; 436-437.

20. Policarpo AJPL. Nucl. Instrum. Methods 1982; 196: 53.

21. Santos FP, dos Santos JMF, Dias THVT, Conde CAN. IEEE Trans. Nucl. Sci. 1995; 42: 611.

22. Policarpo AJPL. Phys. Scr. 1981; 23: 539.

23. Takahashi T, Himi S, Suzuki M, Ruan J-Z, Kubota S. Nucl. Instrum. Methods 1983; 205: 591.

24. Smith A, Favata F, Kowalski TZ. Nucl. Instrum. Methods A 1989; 284: 375.

25. Favata F, Smith A, Badvaz M, Kowalski TZ. Nucl. Instrum. Methods A 1990; 294: 595.

26. Santos FP, Dias THVT, Stauffer AD, Conde CAN. J. Phys. D 1993; 27: 42.

27. Dias THVT, Santos FP, Rachinhas PJBM, Borges FIGM, dos Santos JMF, Conde CAN. J. Appl. Phys. 1999; 85: 303.

28. Borges FIGM, dos Santos JMF, Dias THVT, Santos FP, Rachinhas PJBM, Conde CAN. Nucl. Instrum. Methods A 1999; 422: 321.

29. Veloso JFCA, dos Santos JMF, Conde CAN. Nucl. Instrum. Methods $A$ 2001; 457: 253.

30. Manzo G, Peacock A, Andresen RD, Taylor BG. Nucl. Instrum. Methods 1980; 174: 301.

31. Silva RMC, dos Santos JMF, Conde CAN. Nucl. Instrum. Methods A 1999; 422: 305.

32. Andresen RD, Leimann EA, Peacock A, Taylor BG. IEEE Trans. Nucl. Sci. 1978; 25: 800.

33. Manzo G, Davelaar J, Peacock A, Andresen RD, Taylor BG. Nucl. Instrum. Methods 1980; 177: 595.

34. Erd C, Bavdaz M. In Proceedings of the International Symposium on Optical Science and Engineering - SPIE1992, San Diego, CA, USA,
July 1992. Internal Publication ESLAB 92/094. European Space Research and Technology Centre (ESTEC): Noordwijk.

35. dos Santos JMF, Monteiro CMB, Morgado RE, Conde CAN. Appl. Radiat. Isot. 2000; 53: 739.

36. Torii K, Tsunemi H, Miyata E, Hayashida K. Nucl. Instrum. Methods A 1995; 361: 364.

37. HT 2.2 X-Ray Window. 1999, MOXTEK: OREM, UT.

38. dos Santos JMF, Veloso JFCA, Morgado RE, Conde CAN. Nucl. Instrum. Methods A 1994; 353: 195.

39. Anderson DF, Ku WHM, Mitchel DD, Novick R, Wolf RS. IEEE Trans. Nucl. Sci. 1977; 24: 283.

40. Andresen RD, Leimann EA, Peacock A, Taylor BG, Brownlie G, Stanford P. IEEE Trans. Nucl. Sci. 1977; 24: 810.

41. dos Santos JMF, Bento ACSS, Conde CAN. IEEE Trans. Nucl. Sci. 1992; 39: 541.

42. Goganov DA, Shultz AA, Elkind VB. Instrum. Exp. Tech. (USSR) 1984; 27: 478.

43. dos Santos JMF, Soares AJVD, Monteiro CMB, Morgado RE, Conde CAN. IEEE Trans. Nucl. Sci. 1998; 45: 229.

44. Veloso JFCA, dos Santos JMF, Conde CAN. IEEE Trans. Nucl. Sci. 1995; 42: 369

45. dos Santos JMF, Bento ACSS, Conde CAN. Nucl. Instrum. Methods A 1992; 321: 238.

46. dos Santos JMF, Bento ACSS, Conde CAN. Nucl. Instrum. Methods A 1994; 337: 427.

47. Policarpo AJPL. Nucl. Instrum. Methods 1978; 153: 389.

48. Anderson DF. Nucl. Instrum. Methods 1980; 178: 125.

49. Dangendorf V, Breskin A, Chechik R, Schmidt-Bocking H. Nucl. Instrum. Methods A 1990; 289: 322.

50. Veloso JFCA, dos Santos JMF, Conde CAN. Nucl. Instrum. Methods A 1999; 422: 273.

51. Simons DG, de Korte PAJ, Peacock A, Smith A, Bleeker JAM. IEEE Trans. Nucl. Sci. 1985; 32: 345.

52. de Campos AJ. IEEE Trans. Nucl. Sci. 1984; 31: 133.

53. Veloso JFCA, dos Santos JMF, Conde CAN. Rev. Sci. Instrum. 2000; 71: 2371.

54. Moszynski M, Kapusta M, Zalispka J, Balcerzyk M, Wolski D, Szawlowski M, Klamra W. IEEE Trans. Nucl. Sci. 1999; 46: 243.

55. Lopes JAM, dos Santos JMF, Conde CAN. Nucl. Instrum. Methods A 2000; 454: 421

56. Lopes JAM, dos Santos JMF, Morgado RE, Conde CAN. IEEE Trans. Nucl. Sci. 2000; 47: 928.

57. Veloso JFCA, dos Santos JMF, Conde CAN, Mulhauser F, Knowles P, Donche-Gay C, Huot O, Taqqu D, Kottmann F. Nucl. Instrum. Methods A 2001; 460: 297.

58. Peacock A, Andresen RD, Leimann EA, Long AE, Manzo G, Taylor BG. Nucl. Instrum. Methods 1980; 169: 613.

59. SAES Getters, Milan, www.saesgetters.com, 2001.

60. Silva RMC, Conde CAN. IEEE Trans. Nucl. Sci. 2000; 47: 2075.

61. Simões PCPS, Veloso JFCA, dos Santos JMF, Conde CAN. IEEE Trans. Nucl. Sci. 1997; 44: 521.

62. Simões PCPS, dos Santos JMF, Conde CAN. X-Ray Spectrom. 1997; 26: 182

63. Simões PCPS, dos Santos JMF, Conde CAN. IEEE Trans. Nucl. Sci. 1998; 45: 290

64. Simões PCPS, dos Santos JMF, Conde CAN. Nucl. Instrum. Methods A 1999; 442: 341. 